\title{
DÜBLIN
}

Technological University Dublin ARROW@TU Dublin

2014-06-26

\section{Entropy, Information, Landauer's limit and Moore's law}

\author{
Paul Tobin \\ Technological University Dublin, paul.tobin@tudublin.ie \\ Jonathan Blackledge \\ jonathan.blackledge@tudublin.ie
}

Follow this and additional works at: https://arrow.tudublin.ie/engscheleart

Part of the Computer Sciences Commons

\section{Recommended Citation}

Tobin, P. \& Blackledge, J. (2014). Entropy, information, Landauer's limit and Moore's Law. ISSC 2014, LYIT Limerick, June 26-27

This Conference Paper is brought to you for free and open access by the School of Electrical and Electronic Engineering at ARROW@TU Dublin. It has been accepted for inclusion in Conference papers by an authorized administrator of ARROW@TU Dublin. For more information, please contact arrow.admin@tudublin.ie, aisling.coyne@tudublin.ie,gerard.connolly@tudublin.ie.

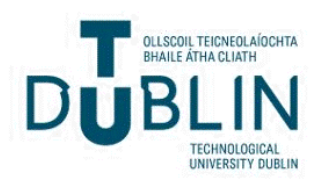




\title{
Entropy, Information, Landauer's limit and Moore's law
}

\author{
P. Tobin, J. Blackledge \\ School of Electrical and Electronic Engineering \\ Dublin Institute of Technology, Ireland
}

E-mail: paul.tobin@dit.ie

jonathan.blackledge@dit.ie

\begin{abstract}
In this paper we explore the link between information and entropy by considering the infamous Maxwell demon thought experiment. A non-rigorous mathematical solution by Leó Szilárd established this link for the first time, as did Claude Shannon nineteen years later. In 1961, Rolf Landauer's mathematical solution resulted in the Landauer limit, which is still being hotly debated, but here we discuss the implication of this limit on Moore's law and future growth in computing power. A workaround the limit is proposed using an Analogue Artificial Neural Network (AANN). Here, we mimic the action of a human brain synapse formed from memristance connected between two Fitzhugh-Nagumo (FN) neuron models. All designs were simulated in Orcad PSpice ${ }^{\circledR}$ version $\mathbf{1 6 . 5}$, but a master-slave synapse was built, tested and outputs compared to simulation results. The synapse was also connected in a star-type network which displayed chaotic-type behaviour for certain parameter values.
\end{abstract}

Keywords - Entropy, information, Maxwell's demon, Landauer limit, Moore's law, memristance, analogue artificial neural networks, synapse, Orcad PSpice, Fitzhugh-Nagumo model.

\section{INTRODUCTION}

If future computer development is to follow Moore's law, alternatives to the von Neumann computer architecture have to be explored. Neuromorphic computing is a relatively new discipline using an AANN to mimic human brain neuronal plasticity. In this paper, an artificial synapse using memristance connected between two electronic neurons, is presented. We concentrate on physical circuits, rather than computer modelling, which brings with it advantages because the system variables are not limited by finite states. Also, circuits present a greater range of interesting behaviours not observed in computer models.

Before we examine neuronal plasticity we must look at the link between entropy and information to justify our proposal in the first instance. Entropy was the name of energy in a system which cannot do useful work. Ludwig Boltzmann said it always increases in any closed system, and engraved on his headstone was $S=k_{B} \ln W$, formulated by the physicist, Willard Gibb. Here, $k_{B}$ $=1.38065156 \times 10^{-23}$ Joules $/ \mathrm{K}$ (value was determined accurately in 2013), is Boltzmann's constant calculated by Max Planck. This is multiplied by the natural log of $W$, where $W$ is an number of equiprobable micro states with the same dimension as entropy.

Figure 1 shows Maxwell's Demon introduced in 1871 by James Clerk Maxwell. The demon observed and separated, high and low energy gas particles into two compartments by opening and closing the gate at opportune times. However, the velocity and temperature of the particles remained unchanged, so when the particles were recombined after separation, it resulted in a temperature drop [1]. This created a paradox in the second law of thermodynamics because entropy cannot be low- 
ered without energy costs.

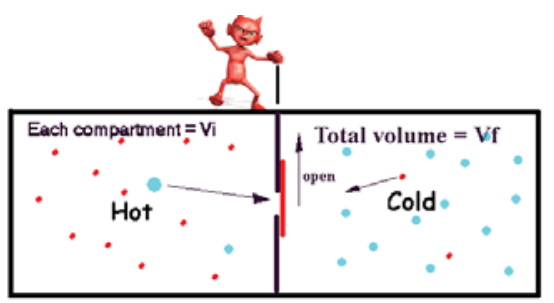

Fig. 1: Maxwell's Demon infamous thought experiment.

Leó Szilárd, a Hungarian electrical engineer, associated entropy with information in his doctoral thesis (1922). A biography by Eugene P. Wigner said Szilárd had a great influence on many scientists including Albert Einstein. In Szilárds' 1929 paper, "On the decrease of entropy in a thermodynamic system by the intervention of intelligent beings" [2], he said information was gathered each time Maxwell's demon opened the gate. This information had entropy that balanced the entropy decrease-paradox solved, almost.

To find an expression for this entropy, consider the work $Q$ done in the thermally-isolated container with pressure $P$ and volume, $V_{f}=2 V_{i}$ :

$Q=\int_{V_{i}}^{V_{f}} P d V=\int_{V_{i}}^{V_{f}} \frac{k_{B} T}{V} d V=k_{B} T \ln 2$

Relating this work with temperture, yields entropy as $\Delta S=\Delta Q / T$, and integrating over the limits, yields $S=-k_{B} \ln 2$.

Claude Shannon, another electrical engineer, also linked entropy and information in his famous 1948 paper "A Mathematical Theory of Communication" [3]. The mathematician, John von Neumann, a friend of Szilárd, said to Shannon he should use the term entropy because "no one knows what entropy is, and in a debate you'll always have the advantage" (Tribus and McIrvine 1971) [4]. In James Gleick's book "The Information" [5], he wrote that this never happened as Shannon was unaware of Szilárd's work! Shannon's entropy for a string of length $n$, is:

$H(x)=-\sum_{i=1}^{n} p\left(x_{i}\right) \ln p\left(x_{i}\right)$ bits

Where $p\left(x_{i}\right)$ is the probability of an event $x_{i}$ occurring from the number of states $n$, each having a probability between 0 and 1 . Shannon's entropy is a measure of the amount of information needed to determine precisely a system state from amongst all possible states. The higher the entropy of a signal, the greater the amount of information it possesses and is also a measure of the unpredictability of a signal. Andrei Kolmogorov, an outstanding Russian mathematician and gifted teacher, in 1959 suggested a modified form of Shannon's entropy, as did Y.Sinai in the same year and is known as the Kolmogorov-Sinai (KS) entropy. Shannon and KS entropies represent the rate at which information is created and is useful for testing whether a time series is chaotic. KS entropy is zero for a regular series, finite for a chaotic series, but infinite for a random signal [6]. Kolmogorov and Ray Solmonoff created Kolmogorov Complexity to specify the minimum length a string of binary digits may be compressed and is essentially the Shannon entropy.

\section{ThE LANDAUER LIMIT}

In lectures on self-replicating machines in 1949, John von Neumann said the minimum energy to operate an electronic switch is zero, provided information is not lost. Similarly, in 1961, Rolf Landauer said the minimum energy to change a computer bit of information is the Landauer limit, equal to $E_{L}=k_{B} T \ln 2=2.87098 \times 10^{-21}$ J $(T=300 \mathrm{~K})$ [7]. In 1982, Charles H. Bennett argued the missing entropy in Maxwell's thought experiment was not due to the information in the demons memory, but erasing it from its memory [8].

The energy for deleting a computer bit of information which produces heat, was measured in 2008 as $E_{b i t}=1 \times 10^{-15} \mathrm{~J}$, which is a million times above the Landauer limit. At the University of Notre Dame, professor George Snider said an integrated circuit operating near the Landauer limit would emit heat per square $\mathrm{cm}$ greater than that from the Sun's surface. However, the Landauer limit ceases when information is not erased, as in reversible computing under adiabatic conditions, but is difficult to implement. Our solution is an AANN which uses memristance as a scaled plastic weight between electronic neurons.

\section{MEMristancE}

Long-lasting brain memories are formed by repeated applications of a stimulus which strengthens the synaptic connection-a process called LongTerm Potentiation (LTP) and AANN systems copy this by modifying its weights between neurons. Professor Leon Chua, in his 1971 paper, proposed a fourth circuit element called memristance, which is resistance with memory. This makes it an ideal candidate as a AANN variable weight storing information as a non-volatile resistive state.

In 2008, Stan Williams in Hewlett-Packard, developed a memristor-type device with a modest switching speed. The device operated in the nanometre range with a potential to achieve packing densities similar to the human brain. In 2010, his team developed a $3 \mathrm{~nm}$ by $3 \mathrm{~nm}$ memristor operating at $1 \mathrm{GHz}$ and with an electron-hole mobil- 
ity of $1 \mathrm{~m} / \mathrm{s}$. This was used in a USB memory key with a performance equalling flash memory technology.

Memristance is formed from a highly-resistive semiconductor, titanium dioxide, which is doped with oxygen atoms and sandwiched between 5 $\mathrm{nm}$ platinum plates. Heating the material removes some negatively-charged oxygen atoms leaving positively-charged oxygen vacancies. These charge carriers are moved by an external bias voltage across the plates, but remain in place once the bias is removed thus making it a non-volatile memory.

Memristors are not commercially available and so we emulated it electronically. Initial investigations simulated memristance in PSpice using three techniques: Netlists, Analogue Behavioural Model (ABM) parts, and electronic emulators [9]. Two memristance models were considered: (i) the linear drift model, which doesn't take into account the distorting effect of electric fields over short nanometre distance, and, (ii) the non-linear drift model, which does.

\section{a) The linear drift model}

Chua related magnetic flux linkage and charge which causes some confusion as these devices do not produce a magnetic flux. However, flux refers to the time integral of the applied voltage and is a function of the charge, $\varphi=f(q)$, so:

$v(t)=\frac{d \varphi}{d t}=\frac{d \varphi}{d q} \frac{d q}{d t}=M(q) \frac{d q}{d t}=M(q) i(t)$

Hence:

$i(t)=\frac{1}{M(q)} v(t)=W(q) v(t)$

Here $W$ is memductance analogous to conductance. The total width of the device is $D$ and $w$, the width of the doped region. When $w$ is zero, the device is off with resistance $R_{o f f}$. However, when the doped region expands such that $w=D$ $=10 \mathrm{~nm}$, then the device is on with resistance $R_{o n}$. The ratio of off to on resistances is $10^{2}$ to $10^{3}$. The normalized width of the doped region with respect to the total width, $D$, is:

$x=\left\{\begin{array}{l}0 \leq(w / D) \leq 1 \\ 0 \text { elsewhere }\end{array}\right.$

The total resistance is the sum of the doped and undoped resistive regions as:

$M(t)=\frac{w(t)}{D} R_{O N}+\left(1-\frac{w(t)}{D}\right) R_{O F F}$

Or, in terms of $x$ :

$M(x)=R_{O F F}-\left(R_{O F F}-R_{O N}\right) x$
For the linear model, the width of the doped region, $w(t)$ is proportional to the charge, $q(t)$ in the device and to the average dopant mobility, $\mu_{v} \approx 10^{-14} m^{-2} s^{-1} V^{-1}$, hence:

$w(t)=\mu_{v} \frac{R_{o n}}{D} q(t)$

The boundary layer speed between the doped and undoped regions is constant and the rate of change of $w(t)$ with time is a function of the current:

$\frac{d w(t)}{d t}=\mu_{v} \frac{R_{\text {on }}}{D} \frac{d q(t)}{d t}=\mu_{v} \frac{R_{\text {on }}}{D} i(t)$

Substituting (9) into (7) and if the on resistance is much less than the off resistance, then:

$M(q)=R_{O F F}\left\{1-\frac{R_{o n}}{\beta} q(t)\right\}$

$\beta=D^{2} / \mu_{v}$, where $D^{2}$ has a much greater effect in the nano scale range.

\section{b) The non-linear drift model}

In the non-linear drift model, small voltages over nano-scale distances, produce vary large electric fields which produce non-linearities in the ionic transport mechanism. This manifests itself at either edge of the doped/undoped regions because the speed of the boundary region gradually decreases to zero at each end. Non-linearity in the Hewlett-Packard model uses a window function $f(x)=\left\{1-(2 x-1)^{2 p}\right\}$, where $p$ is a positive integer between one and ten, as proposed by Joglekar and Wolf (2009) [10]. Figure 2 shows windows for $p=1,4$ and 10 . The inset shows a PSpice ABM1

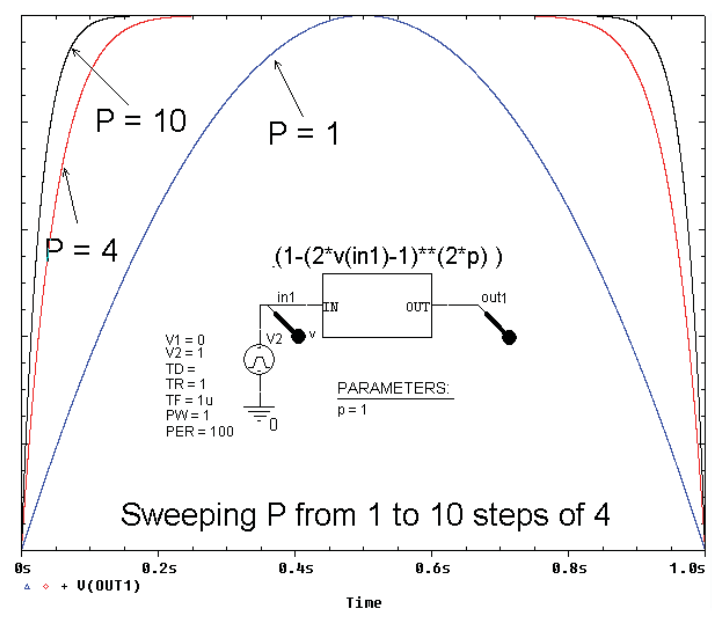

Fig. 2: Joglekar and Wolf windows.

part for displaying the window. The time $\mathrm{x}$-axis represents the normalized width of the memristor, $x=w / D$. Figure 3 displays a pinched hysteresis loop, a bow-tie plot of voltage versus current which 
is similar to the HP bow-tie plot and to that observed in brain synapses. As the voltage increases so does the current. However, as the voltage continues to increase so does the current but to a larger amount because of a change in the memristance. Interestingly, the bow-tie pattern narrows to a line with increasing frequency of the applied signal and the device becomes purely resistive with no memristance. Current and voltage values are

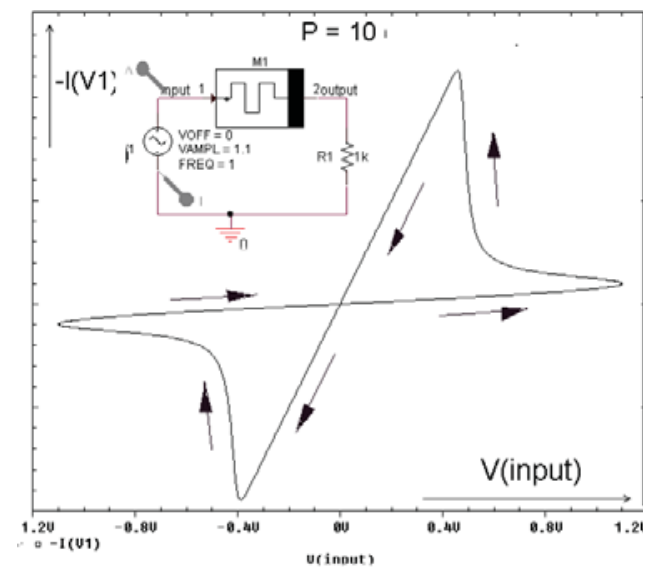

Fig. 3: Bow-tie pattern of $\mathrm{V}$ versus $\mathrm{I}$

zero at the origin indicating power is not dissipated at this point. The memristance symbol in the inset was created and associated with a netlist model which included a window with $p=10$. Memristors are not commercially available so it was necessary to emulate it using the electronic design shown in Figure 4. Here, AD633 4-quadrant multiplier parts implement a cubic polynomial and negative resistance $[11,12]$.

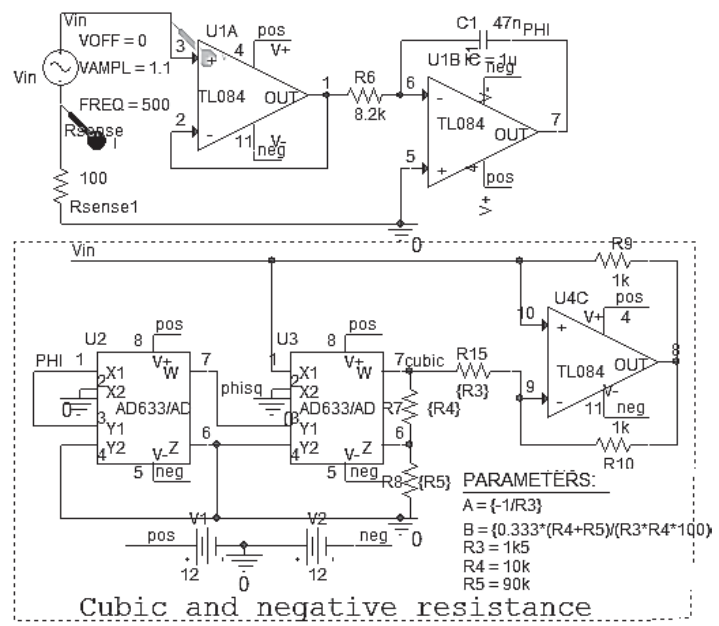

Fig. 4: Linear drift memristance emulator using AD633 IC

Memristors possess resistance which relies on the current flow history, thus a memory can be stored by applying suitable spikes from the neuron. The memristance value (the synaptic connection strength), depends on the polarity, strength and duration of the neuron pulse train. A negative voltage increases the resistance with time, whereas a positive voltage reduces the resistance. There are two learning rules by which synaptic learning occurs between two neurons: One relies on the relative timing between spikes and is called SpikeTiming Dependent Plasticity (STDP), and the second depends on the relative firing rate of the spikes and is called the BCM model after its creators Bienenstock, Cooper and Munro. We now consider an electronic neuron model to generate these voltage impulses.

\section{The Fitzhugh-Nagumo model}

In the human brain, neurons and synapses act as processor and memory, and hence there is no processing bottleneck which results for separate processor and memory units. Neurons communicate using two types of coupling: an inhibiting link to prevents a neuron from firing, and an excitatory link that makes it fire. A short voltage pulse to a neuron produced results similar to that observed in biological neurons and which fires when the voltage amplitude exceeds a threshold.

In 1961, Richard Fitzhugh and J. Nagumo et al, modified the Van der Pol equations to model impulse propagation [13]. The Fitzhugh-Nagumo $(F N)$ model generated voltage impulses similar to the action potential spikes observed in real neurons and the coupled first-order equations are:

$$
\begin{aligned}
& u=-c \int\left(-u+v+\frac{u^{3}}{3}-I(t)\right) d t \\
& v=-\int(-u+b v-a) d t
\end{aligned}
$$

The neuron membrane potential is $u, v$ represents the dynamics of the neuron current, and $I(t)$ is the excitation signal. We replaced the original current excitation signal with a voltage pulse generator with amplitude $0.268 \mathrm{~V}$ and period $2.025 \mathrm{~s}$. The model was simulated initially using ABM parts as shown in Figure 5.

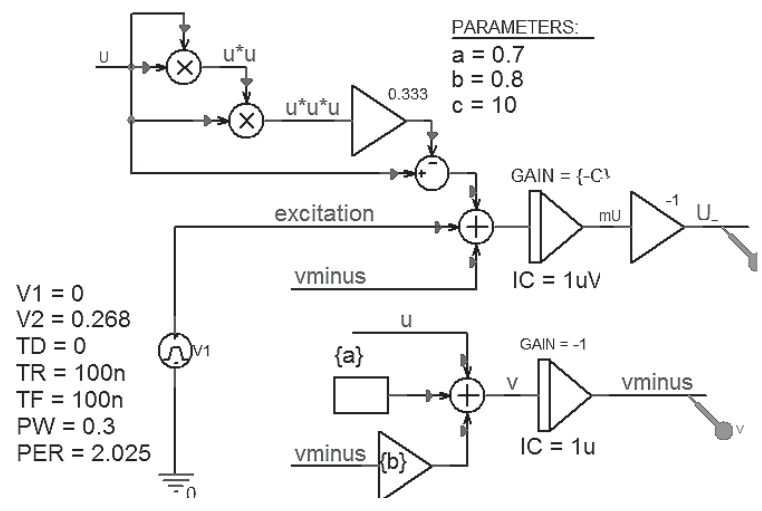

Fig. 5: ABM FN model

Figure 6 shows the $u, v$ and excitation signals over $50 \mathrm{~s}$. The attractor is $u$ on the y-axis and $v$ on 

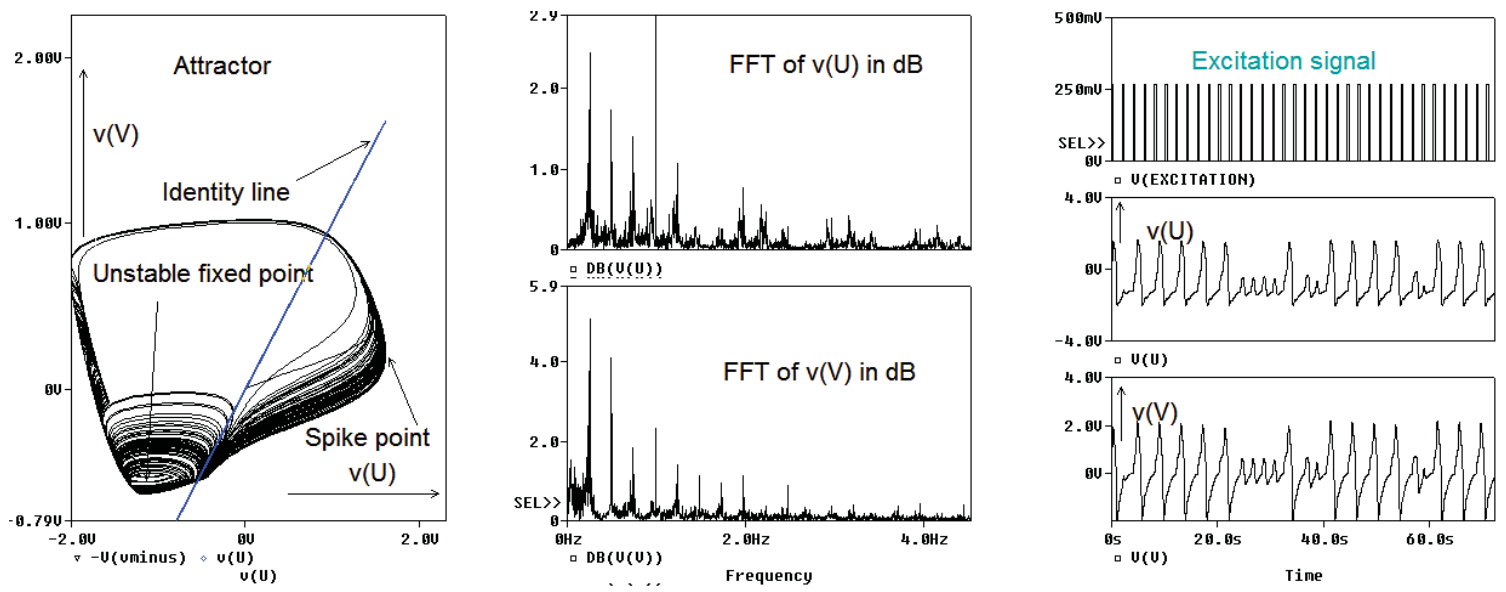

Fig. 6: The FN attractor, FFT spectrum, and excitation and $u$ and $v$ signals.

the $\mathrm{x}$-axis, with the identity line plotted $v$ against itself. An unstable fixed point is observed near the origin. An FN model oscillates periodically when a Hopf bifurcation occurs for excitation amplitudes greater than 0.34. After excitation, a refractory period prevents the neuron from firing. The effect of a range of excitability voltage amplitudes were observed on the limit cycle. The neurons were coupled in a master-slave configuration and also in a star-type network as shown in Figure 7 [13]. Such a network can exhibit chaotic behaviour, depending on the network parameter values. In this arrangement the excitation signal was replaced by the summation of the signals from each neuron and weighted by a positive constant $k$. The equations for $N=6$ are:

$$
\begin{aligned}
& \frac{d u_{i}}{d t}=u_{i}-u_{i}^{3}-v_{i}-\frac{k}{6} \sum_{j=1}^{6} u_{j} \\
& \frac{d v_{i}}{d t}=a+b u_{i}-c v_{i}
\end{aligned}
$$

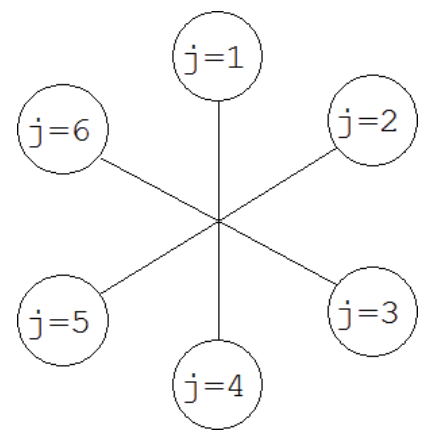

Fig. 7: Star-type neural network

Figure 8 shows the implementation of the FN neuronal model contained in two hierarchical blocks coupled by the resistance, $\mathrm{R} 5$, the memristance. In this master-slave configuration, the memristance value controls the neuron dynamics of the slave neuron and produces frequency locking and chaotic behaviour for certain parameter values. The signals from the actual circuits were very similar to those displayed in Figure 6.

The role of chaos in the brain is still being debated, but its fast response to certain stimuli is probably due to a chaotic element in the signal. Walter J Freeman, a neuroscientist, has championed this belief which remains unproven, but gaining ground $[14,15,16]$. Research indicates the brain reacts extremely fast to situations which threaten it and does so by operating near the edge of criticality (the edge of chaos). For example, prey to avoid being eaten by a predator, will zig-zag chaotically, but without conscious effort. The wide range of the FN FFT spectrum indicates signals of a chaotic nature but this is difficult to prove, even by applying the Lyapunov exponent and KS entropy tests.

\section{Conclusions}

Maxwell's demon thought experiment generated much discussion for nearly a century until Leó Szilárd suggested the missing entropy was the information needed by the demon to keep track of the particles. However, Landauer and Bennett proved that the missing entropy was associated with erasing this information and not in its acquisition. Several scientific experiments by researchers on Maxwell's demon have since verified that information is involved in a solution to the missing entropy [17]. Eventually, Landauer's limit will stop further growth in computing power using the von Neumann architecture unless steps are taken.

Memristance connected between FN electronic neurons might prove fruitful whenever commercial memristors become available. This design produced interesting results that are still being interpreted, but connecting these synapses in more complex networks other than master-slave, is the eventual goal in future research. At the time of submitting this paper, authors Nugent and Molter published a paper in February 2014 on Anti Hebbian and Hebbian (AHaH) computing using mem- 


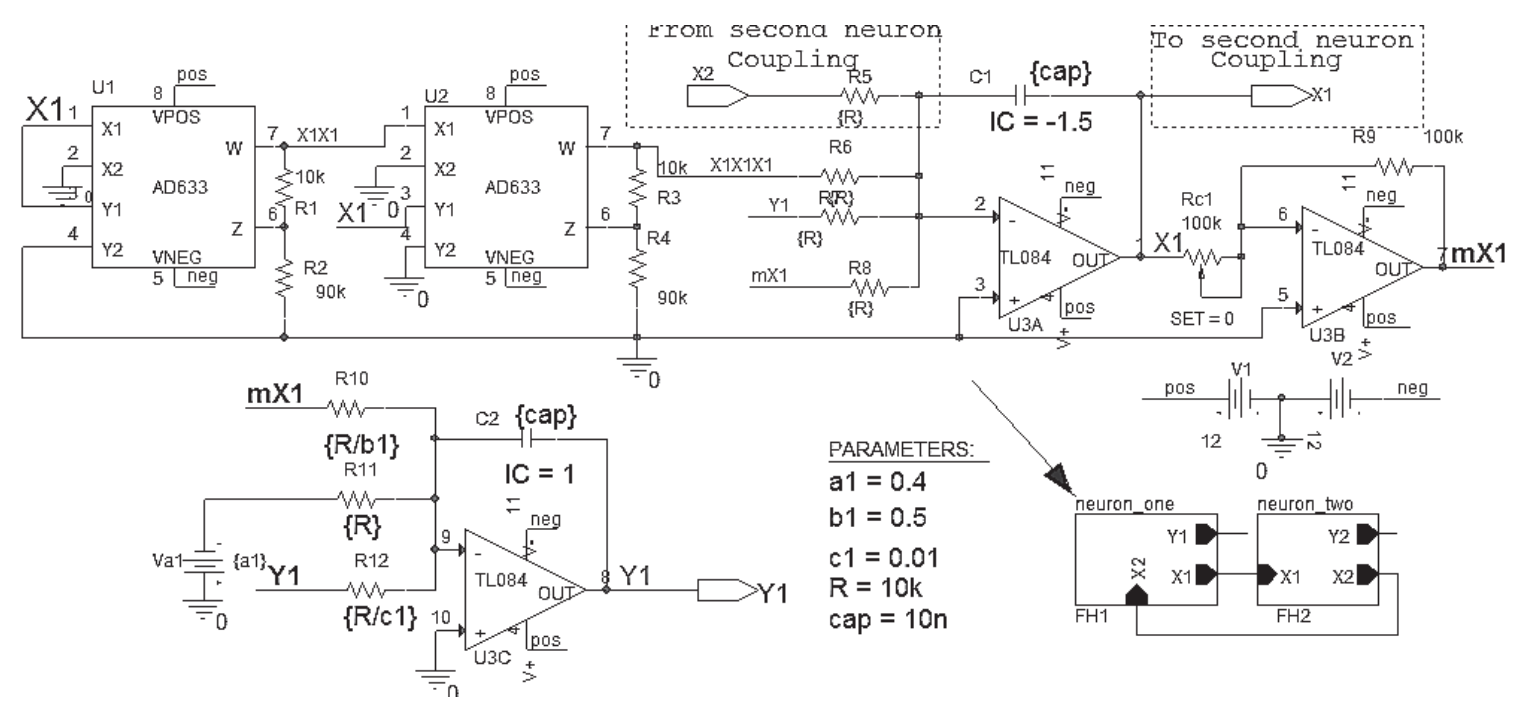

Fig. 8: FN electronic neuron: one of two neurons in a master-slave configuration.

ristance as the variable weight [18].

\section{ACKNOWLEDGements}

The authors are grateful to Dr Marek Rebow at Dublin Institute of Technology for arranging the authors collaborative research programme.

\section{REFERENCES}

[1] John Earman and John D. Norton, "EXORCIST XIV: The wrath of Maxwell's demon. Part I. from Maxwell to Szilárd," Studies In History and Philosophy of Science Part B: Studies in History and Philosophy of Modern Physics 29, 435-471 (1998).

[2] Leó Szilárd, "Uber die Entropieverminderung in einem thermodynamischen System bei Eingriffen intelligenter Wesen," Zeitschrift fur Physik 53, 840-856 (1929), English translation, pp. 110-119.

[3] C. E. Shannon, BA mathematical theory of communication, Bell Syst. Tech. J., vol. 27, pp. 37923 and 62356, 1948.

[4] M. Tribus, E.C. McIrvine, Energy and information, Scientific American, 224 September 1971.

[5] James Gleick, The Information: A History, a Theory, a Flood. New York: Pantheon Books, ISBN 9780375423727, 2011.

[6] Jonathan Blackledge, Cryptography and Steganoraphy: New algorithms and Applications, ISBN 978-83-61993-05-6,2011.

[7] Rolf Landauer, "Irreversibility and heat generation in the computing process," IBM Journal of Research and Development 5, 183-191 (1961).
[8] Charles H. Bennett, "The thermodynamics of 3 computation-a review," International Journal of Theoretical Physics 21, 905-940 (1982).

[9] Paul Tobin, PSpice for Digital Communications Engineering, www.morganclaypool.com ISBN:1598291629, 2007.

[10] Y. Joglekar, S. Wolf, The elusive memristor: properties of basic electrical circuits, (Ml), 124.(2009).

[11] Dalibor Biolek, Z.Biolek, V. Biolkova, SPICE modelling of memristive, memcapacitative and meminductive systems. European Conference on Circuit Theory and Design, (Vc), 2009 .

[12] B. Muthuswamy, "Implementing Memristor Based Chaotic Circuits", IETE Technical Review, VOL. 26, Issue 6, Nov-Dec 2009.

[13] Julien C Sprott, Elegant Chaos: Algebraically Simple Chaotic Flows, Mar 2010.

[14] Walter J Freeman, Scientific American, Vol 264, (2) Pgs. 78-85. February 1991.

[15] http://sulcus.berkeley.edu/flm/ms/ physio.percept.html.

[16] James B. Crutchfield, J. Doyne Farmer, Norman H. Packard and Robert S. Shawl "Chaos," Scientific American, December 1986.

[17] Mark G Raizen, Demons, Entropy, and the Quest for Absolute Zero, Scientific American, 2011.

[18] Nugent MA, Molter TW, AHaH computingfrom metastable switches to attractors to machine learning. PLoS One. Feb 10,2014. 